\title{
A therapeutic insight of carbohydrate and fixed oil from Plantago ovata L. seeds against ketoprofen-induced hepatorenal toxicity in rats
}

\author{
Amal M. El-Feky ${ }^{1}$, Marwa M. Elbatanony ${ }^{1}$, Asmaa F. Aboul Naser² and Manal A. Hamed²*
}

\begin{abstract}
Background: Plantago spp. includes more than 200 species which had been used traditionally to treat many diseases including colds, hepatitis, and infectious diseases. The aim of this study is to evaluate carbohydrates and fixed oil from Plantago ovata L. (Plantaginaceae) seeds against ketoprofen-induced hepatorenal toxicity in rats.

Results: The aqueous extract of P. ovata seeds contain 39\% (wt/wt) carbohydrate as glucose and 35\% (wt/wt) as mucilage. Paper chromatographic analysis and GLC of the mucilage hydrolysate revealed the presence of six free sugars. GC/MS analysis of the saponifiable and unsaponifiable matter of the petroleum ether extract identified 15 compounds from the saponifiable matter. Linoleic acid ethyl ester was the major unsaturated fatty acid, while palmitic acid methyl ester is presented as the major saturated fatty acid. Eighteen compounds were identified from the unsaponifiable matter. 6-Phenyldodecane and 6-phenyl tridecane are presented as major compounds in the unsaponifiable matter. Five steroidal compounds, namely $\beta$-sitosterol, Lupeol, Stigmasterol, Campesterol, and 24(25)dihydrocycloartenol, were identified and confirmed. Carbohydrates and fixed oil administered to normal control rats showed insignificant changes in the oxidative stress markers; liver and kidney function indices, liver DNA degradation pattern, and the histopathological picture of liver and kidney revealed their safety. Ketoprofen induced drastic changes in all the measured parameters. Treatments recorded variable degrees of improvement referring to silymarin as a reference herbal drug.
\end{abstract}

Conclusions: The self-recovery process is not an efficient tool against the ketoprofen toxicity. Treatment with plant carbohydrates exhibited the most potent effect in improving the selected parameters under investigation and served as a safe agent for treatment hepatorenal toxicity in rats.

Keywords: Plantago ovata L., Ketoprofen, Liver, Kidney, Toxicity

\section{Introduction}

Ketoprofen is a non-steroidal anti-inflammatory drug (NSAID) that inhibit cyclooxigenase (COX) enzymes which transforms arachidonic acid to prostaglandin, prostaeyelin, and thromboxane (Tomic et al. 2008). The sustained prostaglandin inhibition leads to sustained reduction in the renal blood flow and thus the renal syndrome ensues. The metabolism of ketoprofen in the liver

\footnotetext{
* Correspondence: manal_hamed@yahoo.com

${ }^{2}$ Department of Therapeutic Chemistry, National Research Centre, 33,

El-Bohouth St., Dokki, Giza, Egypt

Full list of author information is available at the end of the article
}

is intensive, yielding hydrosoluble and liposoluble metabolites. Excessive doses lead to hepatotoxicity and kidney necrosis (Heo et al. 2008; Tomic et al. 2008).

One of the major challenges in clinical application is the detection of new agents that has therapeutic effect against drug-induced hepatorenal toxicity. Many formulations containing the active ingredients of natural products and herbal extracts are used for treatment or even regeneration of hepatic cells (Moradi et al. 2014; Freitag et al. 2015).

Plantago spp. (Plantin) belongs to family Plantaginaceae which includes more than 200 species (Ronsted et al. 2003). It is widely geographic distribution in 
high-temperature regions of the world. Plantago is known to be rich in various secondary metabolites such as phenolic compounds, flavonoids, alkaloids, terpenoids, and vitamin C. These compounds exert antioxidants and anti-inflammatory effect alongside with its high contents of carbohydrate and nutrients (Gill and Valivety 1997). Plantago has been approved as a laxative, antihypercholesterolemic, hypoglycemic, anti-inflammatory, antiviral, analgesic, antioxidant, anticancer, immune modulator, and anti-hypertensive agent (Samuelsen 2000; Haddadian et al. 2014). Plantago has been used traditionally to treat many diseases including colds, hepatitis, skin diseases, infectious diseases, problems related to the digestive organs, respiratory organs, reproduction, and circulation (Beara et al. 2009 and 2010; Chiang et al. 2003; Samuelsen 2000). Plantago ovata leaves, seeds, and husks contain about 76, 78, $58 \%$ polyunsaturated, $21,15,20 \%$ saturated, and 3, 7, 22\% monounsaturated fatty acids, respectively. In addition, the seeds contain mucilaginous compounds which are used as a thickening agent in the pharmaceutical industry for manufacturing tablets (Patel et al. 2016).

The aim of this study is to evaluate the carbohydrate and fixed oil of Plantago ovata seeds against hepatorenal toxicity induced by ketoprofen in rats focusing on carbohydrate and sterols content which may be responsible for the biological activities of Plantago seeds.

\section{Material and methods \\ Chemicals}

All chemicals were of high analytical grade, products of Merck, Germany and Sigma, USA. Ketoprofen was obtained from Sanofi, Egypt, whereas silymarin (hepatoprotective reference drug) was provided from SESDCO, Egypt.

\section{Preparation of the plant extracts}

$P$. ovata seeds were obtained from the local market in March, 2016. The seeds were defatted with petroleum ether $\left(40-60{ }^{\circ} \mathrm{C}\right)$ for preparation of lipoidal extract to obtain the fixed oil, and then macerated in distilled water to prepare the aqueous extract to obtain the carbohydrate. The collected extracts were concentrated in the rotary evaporator $\left(\right.$ at $50{ }^{\circ} \mathrm{C}$ ) and the dried residue was stored at the refrigerator in tight containers until use.

\section{Investigation of carbohydrate profile}

Authentic sugars for paper chromatography (PC) and gas liquid chromatography (GLC) were obtained from Fluka, Switzerland. All solvents used were of pure analytical grade. Quantitative estimation of the total carbohydrate content has been determined as glucose by phenol sulfuric acid method according to Dubois et al. (1956). The mucilage isolated from the aqueous acidified extract according to Laidlow and Percival, (1950). The precipitate was separated by centrifugation, washed with ethanol, stirred with acetone, filtered, and dried in a vacuum desiccators over anhydrous calcium chloride. The nature has been determined according to Evans (1962) and Matz (1962).

Descending paper chromatography analysis of the free sugars and mucilage hydrolysates were carried out on Whatman1MM papers (Whatman Ltd., Maid Stone, Kent, England) using $n$-butanol:acetic acid:water (4:1:5 $\mathrm{v} /$ $v)$ as the developing system. After development, the chromatograms were dried, sprayed with aniline hydrogen phthalate reagent $(0.93 \mathrm{~g}$ aniline and $1.66 \mathrm{~g}$ $O$-phthalic acid dissolved in $100 \mathrm{ml} n$-butanol saturated with water), and heated at $105^{\circ} \mathrm{C}$ for $5 \mathrm{~min}$ (Stahl 1969).

GLC analysis of the mucilage hydrolysates was carried out according to Gertz (1990) on GLC HP 6890; flame ionization detector at $270{ }^{\circ} \mathrm{C}$. The analysis was carried out using ZB-1701 column ( $30 \mathrm{~m} \times 0.25 \mathrm{~m} \times 0.25 \mu \mathrm{m})$, $14 \%$ cyanopropyl phenyl methyl, the carrier gas is helium at flow rate of $1.2 \mathrm{ml} / \mathrm{min}$ under pressure $10.6 \mathrm{psi}$ and velocity of $41 \mathrm{~cm} / \mathrm{s}$. The injector chamber temperature was $-250{ }^{\circ} \mathrm{C}$. Quantitative determination was based on peak area measurement while qualitative identification was carried out by comparison of the retention times of the peaks with those of the authentic sugars.

\section{GC/MS analysis of petroleum ether extract}

Saponification of the petroleum ether extract was performed according to Tsuda et al. (1960). The saponifiable and unsapoifiable matters have been subjected to GC/MS analysis on a model Shimadzu GC/MS-QP5050A. Identification of the constituents has been carried out by comparison of their spectral fragmentation patterns with those of the available database libraries [Wiley (Wiley Int.) USA and NIST (Nat. Inst. St. Technol., USA)] and/or published data (Adams 1989). Quantitative determination was carried out based on peak area integration.

\section{Isolation and identification of the main steroidal compounds from petroleum ether extract}

The conventional column chromatography technique was performed to isolate the main active compounds. Elution was successively carried out by methylene dichloride $\left(\mathrm{CH}_{2} \mathrm{Cl}_{2}\right)$ and increasing the polarity with ethyl acetate. The resulting similar fractions from the column were collected together according to $R_{f}$ values where the isolated compounds were identified by different spectral analyses (IR, mass spectrometry, and $\mathrm{H}^{1}$-NMR). Compound 1 was isolated from $(100 \%$ $\mathrm{CH}_{2} \mathrm{Cl}_{2}$ ), while compounds $2,3,4$, and 5 were isolated from $\mathrm{CH}_{2} \mathrm{Cl}_{2}$ :ethyl acetate $(70: 30 \% v / v),(50: 50 \% v / v)$, $(30: 70 \% v / v),(15: 85 \% v / v)$, respectively. The isolated compounds were further purified several times with preparative TLC technique and then chromatographed on TLC alongside with available authentic references. 


\section{Animals and ethics}

Male Wistar albino rats (100 to $120 \mathrm{~g}$ ) were selected for this study. They were obtained from the Animal House, National Research Center, Egypt. All animals were housed in standard plastic cages in an environmentally controlled condition with free access of water and diet. They were kept 2 weeks for acclimatization before starting any experimental procedures. Anesthetic procedures and handling with animals complied with the ethical guidelines of Medical Ethical Committee of the National Research Center in Egypt to ensure that animals do not suffer at any stage throughout the experiment Approval No. 18211.

\section{Acute toxicity}

Seventy-eight male rats were divided into 13 groups (6 rats each). Each group was orally administered with one oral dose of plant seed oil $(2,4,10,20$, and $40 \mathrm{ml} / \mathrm{kg}$ bode weight) or plant carbohydrates $(40,160,480,640 \mathrm{mg} / \mathrm{kg}$, $1920,3840,7680,13,920 \mathrm{mg} / \mathrm{kg}$ ) and observed for 1 week. The LD50 of both oil and carbohydrates were calculated and $1 / 20$ of each concentration will be selected for further biological determinations. The LD50 of oil and carbohydrates were $40 \mathrm{ml} / \mathrm{kg}$ and $1392.00 \mathrm{mg} / \mathrm{kg}$, respectively. Therefore, the recommended doses were $2 \mathrm{ml} / \mathrm{kg}$ for oil and $696 \mathrm{mg} / \mathrm{kg}$ for carbohydrates.

\section{Experimental design}

Sixty rats were divided into 10 groups (6 rats each).

Group 1: rats were given daily oral dose of $0.5 \mathrm{ml}$ distilled water for 7 days and served as control group. Group 2: rats were given daily oral dose of $0.5 \mathrm{ml}$ plant seed carbohydrates $(696 \mathrm{mg} / \mathrm{kg})$ for 7 days.

Group 3: rats were given daily oral dose of plant seed oil $(2 \mathrm{ml} / \mathrm{kg})$.

Groups 4: rats were given daily oral dose of $0.5 \mathrm{ml}$ ketoprofen for 7 days ( $8 \mathrm{mg} / \mathrm{kg}$ ) (Tomic et al. 2008).

Group5: ketoprofen-induced rats were treated with a daily oral dose of plant seeds carbohydrates.

Groups 6: ketoprofen-induced rats were treated with a daily oral dose of plant seeds oil.

Groups 7: ketoprofen-induced rats were treated with a daily oral dose of $0.5 \mathrm{ml}$ silymarin as a reference drug (100 mg/kg) (Hamed et al. 2012).

Groups 8: recovery; ketoprofen-induced rats and left free for 1 week.

Groups 9: recovery; ketoprofen-induced rats and left free for 2 weeks.

Groups 10: recovery; ketoprofen-induced rats and left free for 3 weeks.

Treatment was carried throughout a period of 1 week after ketoprofen induction.

\section{Sample preparations}

Blood was collected from each animal by puncture of sublingual vein in clean and dry test tubes, left $10 \mathrm{~min}$ at room temperature to clot, and centrifuged at $3000 \mathrm{rpm}$ for serum separation. The separated serum was stored at $-80{ }^{\circ} \mathrm{C}$ for further determinations of liver kidney function tests.

Liver tissue was homogenized in cold $0.9 \mathrm{~N} \mathrm{NaCl}$ (1: 9 $w / v)$ solution, centrifuged at $3000 \mathrm{rpm}$ for $10 \mathrm{~min}$, separated from the supernatant, and stored at $-80{ }^{\circ} \mathrm{C}$ for further antioxidant determinations.

\section{Biochemical assays}

The oxidative stress markers malondialdehyde (MDA), superoxide dismutase (SOD), and glutathione (GSH) were estimated by the methods of Buege and Aust (1978), Nishikimi et al. (1972), and Moron et al. (1979), respectively. Aspartate and alanine aminotransferases (AST, ALT) and alkaline phosphatase (ALP)were estimated by biodiagnostic kit (Biogamma, Stanbio, West Germany) by the method of Gella et al. (1985) and Rosalki et al. (1993), respectively. Urea was determined by the method of Tabacco et al. (1979). Creatinine was measured by the method of Bartels and Böhmer (1971). Total protein was estimated by the method of Bradford (1976).

\section{Histopathological analysis}

Liver and kidney tissues were fixed in $10 \%$ formalin. Paraffin-embedded samples were prepared for sectioning at $4-\mu \mathrm{m}$ thickness. Slides were stained with hematoxylin and eosin and examined by light microscope (Suzuki and Suzuki 1998).

\section{Statistical analysis}

All data were expressed as mean \pm SD of ten rats in each group. Statistical analysis was carried out by one-way analysis of variance (ANOVA), Costat Software Computer Program.

\section{Results}

\section{Investigation of carbohydrate content}

The aqueous extract of $P$. ovata seeds gave positive test for carbohydrate (Molisch 1886), and the total carbohydrate content in this extract was 39\% (wt/wt); calculated as glucose. The percentage of the isolated mucilage was $35 \%$ (wt/wt). No gelatinous precipitate appeared upon reaction with potassium hydroxide; red stain has been obtained with ruthenium red which indicate the mucilaginous nature and eliminate the presence of pectin. Paper chromatographic analysis of the mucilage hydrolysate revealed the presence of galactose, glucose, arabinose, xylose, and riboseandrhamnose as compiled in Table 1. 
Table 1 PC analysis of mucilage hydrolysate in the aqueous extract of $P$. ovata seeds

\begin{tabular}{llll}
\hline Authentic & Developing solvent system & $R_{f}$ with solvent system & Color with aniline phthalate \\
\hline Galacturonic acid & $n$-Butanol-acetic acid-water $(4: 1: 5 \mathrm{~V} / \mathrm{V})$ & 0.11 & Pale brown \\
Glucuronic acid & 0.14 & Pale brown \\
Galactose & 0.16 & Brown \\
Glucose & 0.18 & Brown \\
Fructose & 0.25 & Yellowish brown \\
Arabinose & 0.27 & Brown \\
Xylose & 0.32 & Reddish brown \\
Ribose & 0.36 & Brown \\
Rhamnose & 0.39 & Brown \\
\hline
\end{tabular}

++ : appreciably present, + : present, - : absent

GLC analysis of the mucilage hydrolyzate revealed the presence of six free sugars which represent $96.247 \% \mathrm{wt} /$ wt of the total hydrolysate. Xylose and arabinose are presented as a major sugar $(54.969 \%$ and $31.455 \%$ (wt/wt) of the total hydrolyzate), respectively, in addition to ribose $(1.479 \%)$, rhamnose $(5.482 \%)$, galactose $(2.241 \%)$, and glucose $(0.621 \%)$ as illustrated in Table 2.

\section{GC/MS of the petroleum ether extract}

GC/MS analysis of the saponifiable and unsaponifiable matter of the petroleum ether extract from $P$. ovata seeds has been carried out and identified components are compiled in Tables 3 and 4. Fifteen compounds were identified from the saponifiable matter, representing $89.24 \%$. The saturated fatty acids constituted $23.98 \%$, while the unsaturated fatty acids amounted $65.26 \%$. Linoleic acid ethyl ester $(24.86 \%)$ was the major unsaturated fatty acid, while palmitic acid methyl ester (18.95\%) is presented as the major saturated fatty acid. Eighteen compounds were identified from the unsaponifiable matter amounting to 90.96\%. 6-Phenyldodecane and 6-phenyl tridecane (11.26\% and $11.12 \%$, respectively) are presented as major compounds in the unsaponifiable matter.

\section{Identification of the isolated compounds}

The study led to isolation of five compounds (1-5). Their structure elucidation was confirmed by melting point and some spectral analyses (IR, mass and $\mathrm{H}^{1}$-NMR spectrometry) and comparing with the available authentic.

\section{Compound 1}

$\beta$-Sitosterol in the form of white needles gave positive Salkowski's test and developed a dark violet color with $p$-anisaldehyde. Its $\mathrm{R}_{f}$ was 0.42 . The melting point is $148-149{ }^{\circ} \mathrm{C}$ in agreement to that reported by Kiem et al. (2011) and Awad et al. (2011). The IR spectrum showed absorption bands at $3429 \mathrm{~cm}^{-1}$ (hydroxyl), 2925, 2857, 1650,1460 , and $1039 \mathrm{~cm}^{-1}$ assignable to methylene, $\mathrm{C}=\mathrm{C}$, methyl groups, and $\mathrm{C}-\mathrm{O}$ bond, respectively. Mass spectrum showed $\mathrm{M}^{+}$at $m / z$ 414 (100) for molecular formula $\mathrm{C}_{29} \mathrm{H}_{50} \mathrm{O}$, and other major fragments were at m/z 396 (23), 314 (4), 271 (6), 255 (18), 213 (20), 189 (16), 145 (19), 137 (9), and 57 (78). Comparing the above data with that of previously reported results presented in literature and the $\mathrm{R}_{f}$ value and color under UV with the authentic $\beta$-sitosterol, it could be concluded that the compound is Stigmast-5-en-3-ol ( $\beta$-sitosterol).

\section{Compound 2}

Lupeol was in a form of white crystalline powder; it has melting point $169-170{ }^{\circ} \mathrm{C}$ as stated by Subraya et al. (2013). $\mathrm{R}_{f}$ value: 0.20 , IR spectrum shows absorptions at $3310 \mathrm{~cm}^{-1}$ for $\mathrm{OH}$ group, 2950 and $2860 \mathrm{~cm}^{-1}$ for $\left(\mathrm{CH}_{2}\right.$ and $\mathrm{C}-\mathrm{H}$ stretching), at $1635 \mathrm{~cm}^{-1}$ for $(\mathrm{C}=\mathrm{C}$ str.),

Table 2 GLC analysis of mucilage hydrolysate in the aqueous extract of $P$. ovata seeds

\begin{tabular}{lll}
\hline Authentic sugars & Rt (min.) & Relative percentage (\%) of total mucilage hydrolyzate \\
\hline Arabinose & 8.305 & 31.455 \\
Xylose & 8.609 & 54.969 \\
Ribose & 8.804 & 1.479 \\
Rhamnose & 9.463 & 5.482 \\
Galactose & 13.775 & 2.241 \\
Glucose & 14.514 & 0.621 \\
Total identified sugars & & 96.247 \\
\hline
\end{tabular}


Table 3 GC / MS of the saponifiable matter of $P$. ovata seeds

\begin{tabular}{|c|c|c|c|c|c|}
\hline$R_{t}$ & Percent & Compound & Mol. formula & Mol. weight & $\mathrm{BP}$ \\
\hline 15.90 & 18.95 & Palmitic acid methyl ester & $\mathrm{C}_{17} \mathrm{H}_{34} \mathrm{O}_{2}$ & 270 & 74 \\
\hline 16.06 & 01.31 & 3-Methyl pentadecanoic acid methyl ester & $\mathrm{C}_{17} \mathrm{H}_{34} \mathrm{O}_{2}$ & 270 & 74 \\
\hline 16.23 & 00.56 & 14-Methyl pentadecanoic acid methyl ester & $\mathrm{C}_{17} \mathrm{H}_{34} \mathrm{O}_{2}$ & 270 & 74 \\
\hline 16.30 & 00.33 & 10-Methyl hexadecanoic acid methyl ester & $\mathrm{C}_{18} \mathrm{H}_{36} \mathrm{O}_{2}$ & 284 & 74 \\
\hline 19.75 & 00.60 & 10,12-Octadecadienoic acid methyl ester & $\mathrm{C}_{19} \mathrm{H}_{34} \mathrm{O}_{2}$ & 294 & 67 \\
\hline 19.92 & 07.80 & 9-Octadecenoic acid methyl ester & $\mathrm{C}_{19} \mathrm{H}_{36} \mathrm{O}_{2}$ & 296 & 55 \\
\hline 20.41 & 08.91 & Octadecenoic acid methyl ester & $\mathrm{C}_{19} \mathrm{H}_{38} \mathrm{O}_{2}$ & 298 & 74 \\
\hline 23.44 & 01.21 & 11-Nonadecenoic acid methyl ester & $\mathrm{C}_{20} \mathrm{H}_{38} \mathrm{O}_{2}$ & 310 & 55 \\
\hline 24.16 & 01.56 & 13-Nonadecenoic acid methyl ester & $\mathrm{C}_{20} \mathrm{H}_{38} \mathrm{O}_{2}$ & 310 & 55 \\
\hline 24.54 & 24.86 & Linoleic acid ethyl ester & $\mathrm{C}_{20} \mathrm{H}_{36} \mathrm{O}_{2}$ & 308 & 67 \\
\hline 24.69 & 02.09 & Arachidonic acid methyl ester & $\mathrm{C}_{21} \mathrm{H}_{42} \mathrm{O}_{2}$ & 326 & 74 \\
\hline 26.84 & 00.38 & Heneicosanoic acid methyl ester & $\mathrm{C}_{22} \mathrm{H}_{44} \mathrm{O}_{2}$ & 340 & 74 \\
\hline 28.21 & 01.61 & 13-Docosenoic acid methyl ester & $\mathrm{C}_{23} \mathrm{H}_{44} \mathrm{O}_{2}$ & 352 & 55 \\
\hline 28.69 & 02.83 & Docosanoic acid methyl ester & $\mathrm{C}_{23} \mathrm{H}_{46} \mathrm{O}_{2}$ & 354 & 74 \\
\hline 29.11 & 16.24 & 15-Tetracosenoic acid methyl ester & $\mathrm{C}_{25} \mathrm{H}_{48} \mathrm{O}_{2}$ & 380 & 55 \\
\hline \multicolumn{2}{|c|}{ Saturated fatty acids } & & $23.98 \%$ & & \\
\hline \multicolumn{2}{|c|}{ Unsaturated fatty acids } & & $65.26 \%$ & & \\
\hline \multicolumn{2}{|c|}{ Total identified fatty acids } & & $89.24 \%$ & & \\
\hline
\end{tabular}

$1460 \mathrm{~cm}^{-1}(\mathrm{C}-\mathrm{H}), 1037 \mathrm{~cm}^{-1}$ for $(\mathrm{C}-\mathrm{O}$ stretching of secondary alcohol). Mass spectrum gave $\mathrm{M}^{+}$at $m / z 426$ for molecular formula $\mathrm{C}_{30} \mathrm{H}_{50} \mathrm{O}$ and base peak at 43 (100). The other fragments are at $m / z 382(10), 367$ (8), 261 (15), 213 (17), 161 (20), 408 (13), 393 (13), 365 (18), 297

Table 4 GC / MS of the unsaponifiable matter of $P$. ovata seeds

\begin{tabular}{llllll}
\hline$R_{\mathrm{t}}$ & Percent & Compound & Mol. formula & Mol. weight & $\mathrm{BP}$ \\
\hline 22.07 & 1.74 & 6-Phenyl undecane & $\mathrm{C}_{17} \mathrm{H}_{28}$ & 232 & 91 \\
22.16 & 5.69 & 5-Phenyl undecane & $\mathrm{C}_{17} \mathrm{H}_{28}$ & 232 & 91 \\
22.44 & 3.53 & 4-Phenyl undecane & $\mathrm{C}_{17} \mathrm{H}_{28}$ & 232 & 91 \\
22.99 & 2.61 & 3-Phenyl undecane & $\mathrm{C}_{17} \mathrm{H}_{28}$ & 232 & 91 \\
23.94 & 5.26 & 2-Phenyl undecane & $\mathrm{C}_{17} \mathrm{H}_{28}$ & 232 & 105 \\
24.41 & 11.26 & 6-Phenyl dodecane & $\mathrm{C}_{18} \mathrm{H}_{30}$ & 246 & 91 \\
24.52 & 10.93 & 5-Phenyl dodecane & $\mathrm{C}_{18} \mathrm{H}_{30}$ & 246 & 91 \\
24.83 & 7.58 & 4-Phenyl dodecane & $\mathrm{C}_{18} \mathrm{H}_{30}$ & 246 & 91 \\
25.36 & 4.87 & 3-Phenyl dodecane & $\mathrm{C}_{18} \mathrm{H}_{30}$ & 246 & 91 \\
26.30 & 6.45 & 2-Phenyl dodecane & $\mathrm{C}_{18} \mathrm{H}_{30}$ & 246 & 105 \\
26.67 & 11.12 & 6-Phenyl tridecane & $\mathrm{C}_{19} \mathrm{H}_{32}$ & 260 & 91 \\
26.84 & 6.99 & 5-Phenyl tridecane & $\mathrm{C}_{19} \mathrm{H}_{32}$ & 260 & 91 \\
27.15 & 5.19 & 4-Phenyl tridecane & $\mathrm{C}_{19} \mathrm{H}_{32}$ & 260 & 91 \\
27.72 & 2.80 & 3-Phenyl tridecane & $\mathrm{C}_{19} \mathrm{H}_{32}$ & 260 & 91 \\
28.72 & 4.27 & 2-Phenyl tridecane & $\mathrm{C}_{19} \mathrm{H}_{32}$ & 260 & 105 \\
34.27 & 0.21 & Ergosterol & $\mathrm{C}_{28} \mathrm{H}_{44} \mathrm{O}$ & 396 & 69 \\
37.76 & 0.25 & 3-Sitosterol & $\mathrm{C}_{29} \mathrm{H}_{50} \mathrm{O}$ & 414 & 41 \\
50.01 & 0.21 & Isoadiantol B & $\mathrm{C}_{29} \mathrm{H}_{50} \mathrm{O}$ & 414 & 191 \\
Total identified compounds & & $90.96 \%$ & \\
\hline
\end{tabular}

(14), 218 (85), 203 (43), 189 (50), 175 (41), 161 (40), 147 (49), 135 (65), 121 (70), 95 (82), 81 (35), 69 (70), 55 (95), and $41(65) \cdot \mathrm{H}^{1}$-NMR spectrum showed the following signals: at $\delta 4.70(\mathrm{~s}, 1 \mathrm{H}, \mathrm{H}-29), \delta 4.55(\mathrm{~s}, 1 \mathrm{H}, \mathrm{H}-27), \delta$ $3.20(\mathrm{~d}, 1 \mathrm{H}, \mathrm{H}-3), \delta 2.35(\mathrm{~m}, 1 \mathrm{H}, \mathrm{H}-19), \delta 1.90(\mathrm{~m}, 1 \mathrm{H}$, $\mathrm{H}-21), \delta 1.70(\mathrm{~s}, 3 \mathrm{H}, \mathrm{H}-30), \delta 1.68(\mathrm{t}, 1 \mathrm{H}, \mathrm{H}-13), \delta 1.60$ (s, 3H, H-2a), $\delta 1.55$ (s, 3H, H-2b), $\delta 1.53$ (d, 1H, H-11), $\delta 1.44(\mathrm{~d}, 1 \mathrm{H}, \mathrm{H}-14), \delta 1.40(\mathrm{q}, 1 \mathrm{H}, \mathrm{H}-6), \delta 1.34(\mathrm{~s}, 1 \mathrm{H}$, $\mathrm{H}-21), \delta 1.30$ (q, 1H, H-12), $\delta 1.27$ (s, 1H, H-9), $\delta 1.05$ (s, $1 \mathrm{H}, \mathrm{H}-23), \delta 1.00$ (d, 1H, H-15), $\delta 0.98$ (s, 3H, H-23), $\delta 0.95$ (s, 1H, H-27), $\delta 0.90$ (t, 1H, H-18), $\delta 0.85$ (s, 3H, $\mathrm{H}-25), \delta 0.78$ (s, 3H, H-28), $\delta 0.67$ (d, 1H, H-5).

\section{Compound 3}

Stigmasterol was obtained as white crystalline powder with melting point $169-170{ }^{\circ} \mathrm{C}$ which is in agreement with that mentioned by Subraya et al. (2013). $R_{f}$ value recorded 0.20, and the IR spectrum showed absorptions at $3400 \mathrm{~cm}^{-1}$ for $\mathrm{OH}$ group, 2919 and $2800 \mathrm{~cm}^{-1}$ for $\left(\mathrm{CH}_{2}\right.$ and $\mathrm{C}-\mathrm{H}$ stretching), at $1630 \mathrm{~cm}^{-1}$ and $1627 \mathrm{~cm}^{-1}$ for $\left(\mathrm{C}=\mathrm{C}\right.$ str.), $1457 \mathrm{~cm}^{-1}(\mathrm{C}-\mathrm{H}), 1057 \mathrm{~cm}^{-1}$ for $(\mathrm{C}-\mathrm{O}$ stretching of secondary alcohol). Finally, 625 (rocking vibration of $\mathrm{CH}_{2}$ ). Mass spectrum gives $\mathrm{M}^{+}$at $m / z 412$ for molecular formula $\mathrm{C}_{29} \mathrm{H}_{48} \mathrm{O}$ and 55 (100) for the base peak. The other fragments at $m / z 394$ (20), 255 (2), 213 
(6), 199 (4), 159 (10), 145 (13), 133 (18), 121 (23), 105 (28), 91 (40), 83 (48), 81 (76), and 69 (56). $\mathrm{H}^{1}$-NMR spectrum recorded the following signals at $\delta 0.87$ to $\delta 1.1$ $(\mathrm{m}, 18 \mathrm{H}, 6 \mathrm{xCH} 3)$; at $\delta 1.1$ to $\delta 1.20(\mathrm{~m}, 18 \mathrm{H}, 9 \times \mathrm{CH} 2)$, $(8 \mathrm{H}$, methine protons)appeared at $\delta 1.8$ to $\delta 2.3$, while $(1 \mathrm{H}, \mathrm{CHOH})$ appeared at $\delta 3.5$; furthermore, the signal at $\delta 5.73$ attributed to oleifinic hydrogen $\mathrm{H}-4$ in the steroidal nucleus. Signals at $\delta 5.02$ and $5.15(d d, J=8.6$ and $15.2 \mathrm{~Hz}$ ) assigned to methine protons $\mathrm{H}-22$ and $\mathrm{H}-23$; and at $\delta 5.34(d, 5.2 \mathrm{~Hz})$ to $\mathrm{H}-6$.

\section{Compound 4}

Campesterol was in a form of white amorphous powder with melting point $157-158{ }^{\circ} \mathrm{C}$ as stated by Choi et al. (2007) and Jain and Bari (2010). Its $R_{f}=0.73$. IR showed absorption bands at 3428.79 (hydroxyl) $\mathrm{cm}^{-1}, 2924.22$, 2857.67, 1650.86, 1460.23, and $1039.59 \mathrm{~cm}^{-1}$ assignable to methylene, $\mathrm{C}=\mathrm{C}$, methyl groups, and $\mathrm{C}-\mathrm{O}$ bond, respectively. Mass spectrum gave $\mathrm{M}^{+}$at $m / z 400$ for a molecular formula $\mathrm{C}_{28} \mathrm{H}_{48} \mathrm{O}$ with other significant mass spectral peaks at $m / z$ values 75 (6), 129 (100), 135 (2), 187 (1), 213 (12), 227 (5), 255 (2), 261 (1), 315 (2), 343 (9), 367 (3), and $382(2) . \mathrm{H}^{1}$-NMR shows that $\mathrm{H}-3$ proton appeared at $\delta 3.21$ as triplet of doublet, $\mathrm{H}-6$ oleifinic proton showed a multiple et at $\delta 5.10$ owing to the double bond between C-5 and C- 6 and six methyl protons appeared at $\delta 1.27, \delta 1.14, \delta 1.09, \delta 1.00, \delta 0.98$, and $\delta 0.95$ singlets for the methyl groups at $\mathrm{H}-18$ and $\mathrm{H}-19$. Doublets at $\delta 1.01$,
$0.82,0.80$ and a triplet at 0.84 confirm the presence of C-21, C-26, C-27, and C-29 methyl groups.

\section{Compound 5}

24(25)-Dihydrocycloartenol was in a form of white crystalline powder, melting point $106-107^{\circ} \mathrm{C}$ in agreement with Pascal et al. (1993). The $\mathrm{H}^{1-} \mathrm{NMR}$ spectrum assured the presence of signals at $\delta 0.341(\mathrm{lH}, \mathrm{d}, J=4.0, \mathrm{H} 19$ exo), $\delta$ $0.562(\mathrm{lH}, \mathrm{d}, J=3.9, \mathrm{H} 19$ endo), $\delta 0.791$ (3H, s, H28), $\delta$ $0.791(3 \mathrm{H}, \mathrm{d}, J=7, \mathrm{H} 21), \delta 0.825(3 \mathrm{H}, \mathrm{d}, J=6.6, \mathrm{H} 26$ or H27), $\delta 0.861$ (3H, d, $J=6.6, \mathrm{H} 26$ or H27), $\delta 0.912(3 \mathrm{H}, \mathrm{s}$, H30), $\delta 0.958$ (6H, s, H18 and H29), $\delta 3.195(\mathrm{lH}, \mathrm{dd}, J=10$, $J=5, \mathrm{H} 3 \alpha)$. Mass spectrum gave $\mathrm{M}^{+}$at $m / z 428$ for molecular formula $\mathrm{C}_{30} \mathrm{H}_{62} \mathrm{O}$. The other fragments were recorded at $m / z 428$ (33), 413 (66), 410 (65), 395 (100), 367 (35), 341 (49), 315 (34), 288 (94).

\section{Oxidative stress markers}

Nonsignificant changes were observed in oxidative stress markers after administration of normal rats with plant seed oil and carbohydrates. Rats administered ketoprofen showed significant decrease in SOD and GSH levels compared with the control group, while significant increase in MDA level was observed. Ketoprofen-induced rats treated with plant seeds oil and carbohydrates recorded significant increase in SOD and GSH levels as compared by the ketoprofen group, while significant decrease in MDA level was noticed (Table 5). Therefore, treatments with plant seeds carbohydrate, seeds oil, and

Table 5 Therapeutic effect of $P$. ovata seed carbohydrates and fixed oil on hepatic oxidative stress markers of ketoprofen toxicity in rats

\begin{tabular}{|c|c|c|c|}
\hline Groups & SOD ( $\mu \mathrm{g} / \mathrm{mg}$ protein) & GSH ( $\mu \mathrm{g} / \mathrm{gm}$ tissue) & MDA ( $\mu \mathrm{mol} / \mathrm{mg}$ protein) \\
\hline Control & $18.17^{\mathrm{a}} \pm 1.77$ & $11.87^{\mathrm{a}} \pm 1.36$ & $\begin{array}{l}1.53^{\mathrm{e}} \pm 0.26 \\
-\end{array}$ \\
\hline P. ovata seed carbohydrates & $\begin{array}{l}16.90^{\mathrm{ab}} \pm 2.28 \\
(-6.98)\end{array}$ & $\begin{array}{l}10.50^{\mathrm{b}} \pm 0.94 \\
(-11.54)\end{array}$ & $\begin{array}{l}1.59^{\text {de }} \pm 0.14 \\
(+3.92)\end{array}$ \\
\hline P. ovata seeds oil & $\begin{array}{l}17.50^{\mathrm{ab}} \pm 2.04 \\
(-3.60)\end{array}$ & $\begin{array}{l}10.30^{b} \pm 0.56 \\
(-13.22)\end{array}$ & $\begin{array}{l}1.92^{\text {cde }} \pm 0.43 \\
(+25.49)\end{array}$ \\
\hline Ketoprofen & $\begin{array}{l}8.00^{d} \pm 1.91 \\
(-55.97)\end{array}$ & $\begin{array}{l}4.68^{e} \pm 0.57 \\
(-60.57)\end{array}$ & $\begin{array}{l}4.70^{\mathrm{a}} \pm 0.70 \\
(+207.18)\end{array}$ \\
\hline $\begin{array}{l}\text { Ketoprofen }+ \\
\text { P. ovata seed carbohydrates }\end{array}$ & $\begin{array}{l}13.97^{b c} \pm 1.16 \\
{[+74.67]}\end{array}$ & $\begin{array}{l}9.20^{\mathrm{bc}} \pm 0.30 \\
{[+96.58]}\end{array}$ & $\begin{array}{l}2.32^{\text {cde }} \pm 0.37 \\
{[-49.56]}\end{array}$ \\
\hline $\begin{array}{l}\text { Ketoprofen }+ \\
\text { P. ovata seeds oil }\end{array}$ & $\begin{array}{l}12.50^{\mathrm{bc}} \pm 1.87 \\
{[+56.25]}\end{array}$ & $\begin{array}{l}8.50^{c d} \pm 0.73 \\
{[+81.62]}\end{array}$ & $\begin{array}{l}2.56^{\mathrm{bc}} \pm 0.73 \\
{[-44.34]}\end{array}$ \\
\hline $\begin{array}{l}\text { Ketoprofen + } \\
\text { Silymarin. }\end{array}$ & $\begin{array}{l}14.85^{\mathrm{abc}} \pm 1.75 \\
{[+85.63]}\end{array}$ & $\begin{array}{l}8.70^{\text {cd }} \pm 1.18 \\
{[+85.89]}\end{array}$ & $\begin{array}{l}1.94^{\text {cde }} \pm 0.61 \\
{[-57.82]}\end{array}$ \\
\hline Ketoprofen left 1 week for recovery & $\begin{array}{l}8.07^{d} \pm 2.44 \\
{[+0.80]}\end{array}$ & $\begin{array}{l}7.35^{d} \pm 0.40 \\
{[+57.05]}\end{array}$ & $\begin{array}{l}3.18^{b} \pm 0.25 \\
{[-30.86]}\end{array}$ \\
\hline Ketoprofen left 2 weeks for recovery & $\begin{array}{l}9.10^{d} \pm 1.27 \\
{[+13.75]}\end{array}$ & $\begin{array}{l}7.83^{c d} \pm 0.40 \\
{[+67.30]}\end{array}$ & $\begin{array}{l}2.67^{b c} \pm 0.29 \\
{[-41.95]}\end{array}$ \\
\hline Ketoprofen left 3 weeks for recovery & $\begin{array}{l}11.85^{\complement} \pm 1.59 \\
{[+48.12]}\end{array}$ & $\begin{array}{l}8.10^{\text {cd }} \pm 0.48 \\
{[+73.07]}\end{array}$ & $\begin{array}{l}2.45^{\mathrm{bcd}} \pm 0.32 \\
{[-46.73]}\end{array}$ \\
\hline
\end{tabular}

Data are mean \pm SD of six rats/group.

Groups having the same letters are non-significantly different, while those having different letters are significantly different at $p<0.05$

Values between brackets are \% changes over control group

Values between parentheses are \% changes over ketoprofen group 
silymarin showed improvement in SOD level by 32.86 , 24.78 , and $37.69 \%$, respectively. GSH improved by 38.07 , 32.18 , and $33.86 \%$, respectively, while MDA was improved by $149.20,133.33$, and $173.85 \%$, respectively. In auto-healing ketoprofen groups, we noticed a positive correlation between these parameters and the time of auto-recovery, but not reached to the treatment degree (Fig. 1a).

\section{Liver function enzymes}

Regarding to the liver function enzymes, insignificant changes were observed in AST, ALT and ALP after administration of normal rats with plant seed oil and carbohydrates. Rats administered with ketoprofen showed significant increase in AST, ALT, and ALP levels compared with the control group. Ketoprofen-induced rats treated with plant seeds oil and carbohydrates recorded significant decrease in AST, ALT, and ALP levels as compared by the ketoprofen group (Table 6). Treatments with plant seeds carbohydrate, seeds oil, and silymarin showed amelioration in AST level by 137.66, 120.21, and $132.94 \%$, respectively. ALT was improved by 145.39 , 94.39, and $177.05 \%$, respectively. ALP was improved by $46.16,24.32$, and $31.76 \%$, respectively. In the auto-recovery ketoprofen groups, the liver function indices were improved to some extent by time (Fig. 1b).

\section{Liver DNA fragmentation pattern}

High degree of DNA fragmentation pattern was observed in Table 7 and Fig. 2 represented by significant increase in tailed DNA, DNA tail-length, total DNA, and DNA tail moment. In addition, significant decrease in un-tailed DNA was noticed in ketoprofen-induced liver toxicity group as compared with the control group. Treatment with plant seeds carbohydrate, oils, and silymarin drug showed variable degrees of improvement in
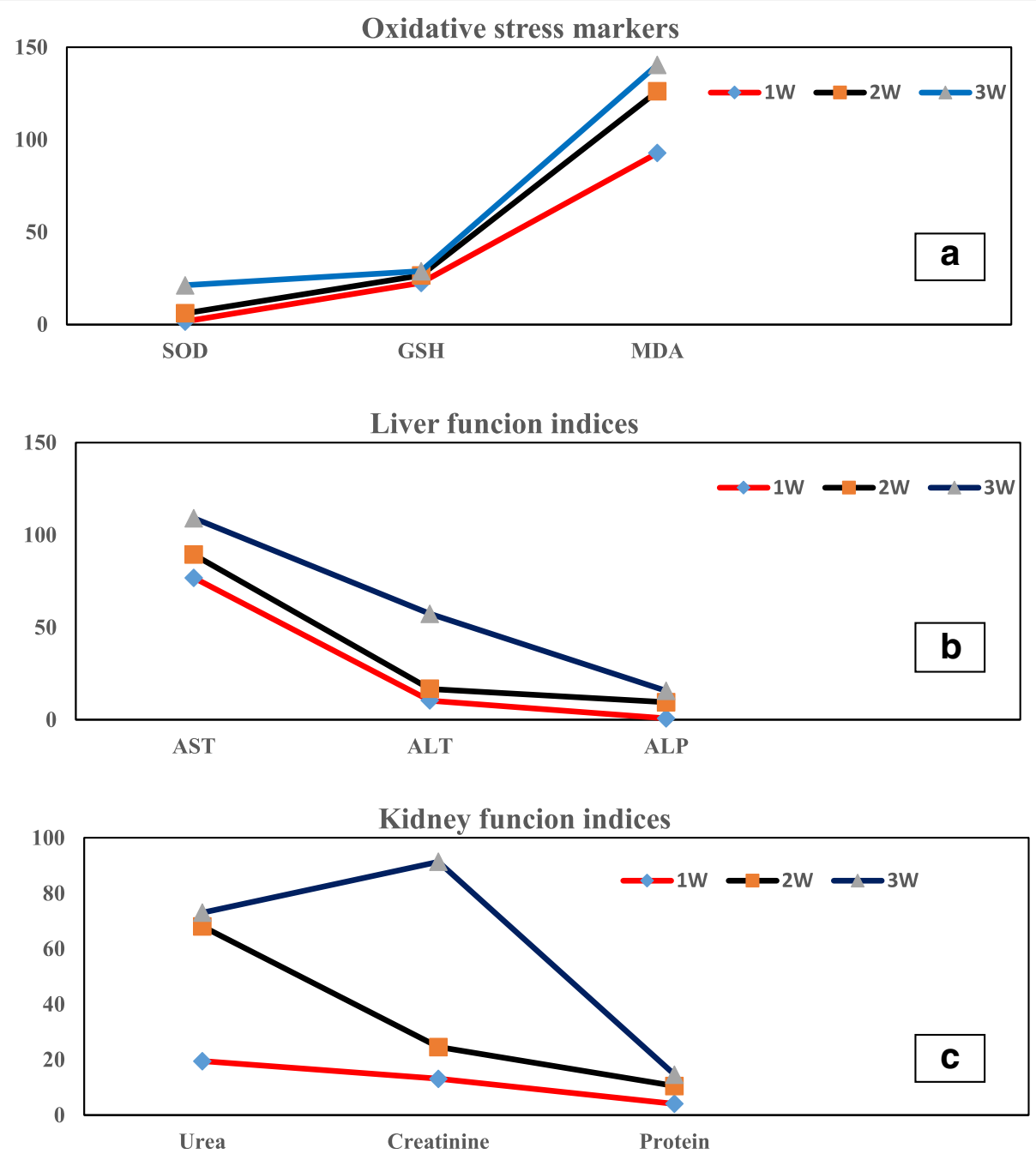

Fig. 1 Recovery percentages of oxidative stress markers, liver function enzymes, and kidney function indices in ketoprofen-treated rats left free without treatment for one (a), two (b), and three (c) weeks 
Table 6 Therapeutic effect of $P$. ovata seed carbohydrates and fixed oil on liver function enzymes of ketoprofen toxicity in rats

\begin{tabular}{|c|c|c|c|}
\hline Groups & AST(Unit/L) & ALT(Unit/L) & ALP (Unit/L) \\
\hline Control & $53.73^{\mathrm{e}} \pm 3.88$ & $17.82^{\mathrm{e}} \pm 3.38$ & $125.93^{f} \pm 5.43$ \\
\hline P. ovata seed carbohydrates & $\begin{array}{l}56.17^{\mathrm{e}} \pm 2.66 \\
(+4.54)\end{array}$ & $\begin{array}{l}21.90^{\mathrm{e}} \pm 1.28 \\
(+22.89)\end{array}$ & $\begin{array}{l}134.57^{f} \pm 6.86 \\
(+6.86)\end{array}$ \\
\hline P. ovata seeds oil & $\begin{array}{l}62.02^{e} \pm 2.20 \\
(+15.43)\end{array}$ & $\begin{array}{l}22.30^{e} \pm 2.69 \\
(+25.14)\end{array}$ & $\begin{array}{l}140.81^{f} \pm 8.05 \\
(+11.81)\end{array}$ \\
\hline Ketoprofen & $\begin{array}{l}147.40^{\mathrm{a}} \pm 9.16 \\
(+174.23)\end{array}$ & $\begin{array}{l}63.32^{\mathrm{a}} \pm 2.85 \\
(+255.33)\end{array}$ & $\begin{array}{l}221.56^{\mathrm{a}} \pm 10.96 \\
(+75.93)\end{array}$ \\
\hline $\begin{array}{l}\text { Ketoprofen }+ \\
\text { P. ovata seed carbohydrates }\end{array}$ & $\begin{array}{l}73.43^{d} \pm 7.67 \\
{[-50.18]}\end{array}$ & $\begin{array}{l}37.41^{d} \pm 6.07 \\
{[-40.91]}\end{array}$ & $\begin{array}{l}163.43^{e} \pm 6.23 \\
{[-26.23]}\end{array}$ \\
\hline $\begin{array}{l}\text { Ketoprofen }+ \\
\text { P. ovata seeds oil }\end{array}$ & $\begin{array}{l}82.81^{c d} \pm 8.15 \\
{[-43.81]}\end{array}$ & $\begin{array}{l}46.50^{\complement} \pm 4.89 \\
{[-26.56]}\end{array}$ & $\begin{array}{l}190.93^{\mathrm{cd}} \pm 11.05 \\
{[-13.82]}\end{array}$ \\
\hline $\begin{array}{l}\text { Ketoprofen + } \\
\text { Silymarin. }\end{array}$ & $\begin{array}{l}75.94^{d} \pm 4.44 \\
{[-48.48]}\end{array}$ & $\begin{array}{l}31.77^{d} \pm 3.48 \\
{[-49.82]}\end{array}$ & $\begin{array}{l}181.56^{d} \pm 17.71 \\
{[-18.05]}\end{array}$ \\
\hline Ketoprofen left 1 week for recovery & $\begin{array}{l}106.16^{b} \pm 10.19 \\
{[-27.97]}\end{array}$ & $\begin{array}{l}61.47^{\mathrm{a}} \pm 5.68 \\
{[-2.92]}\end{array}$ & $\begin{array}{l}220.62^{\mathrm{a}} \pm 7.73 \\
{[-0.42]}\end{array}$ \\
\hline Ketoprofen left 2 weeks for recovery & $\begin{array}{l}99.43^{b} \pm 7.67 \\
{[-32.54]}\end{array}$ & $\begin{array}{l}60.35^{\mathrm{a}} \pm 4.73 \\
{[-4.70]}\end{array}$ & $\begin{array}{l}209.68^{\mathrm{ab}} \pm 7.93 \\
{[-5.36]}\end{array}$ \\
\hline Ketoprofen left 3 weeks for recovery & $\begin{array}{l}88.81^{c} \pm 9.08 \\
{[-39.74]}\end{array}$ & $\begin{array}{l}53.11^{b} \pm 5.51 \\
{[-16.12]}\end{array}$ & $\begin{array}{l}201.87^{\mathrm{bc}} \pm 10.82 \\
{[-8.88]}\end{array}$ \\
\hline
\end{tabular}

Data are mean \pm SD of six rats/group

Groups having the same letters are non- significantly different, while those having different letters are significantly different at $p<0.05$

Values between brackets are \% changes over control group

Values between parentheses are \% changes over ketoprofen group

DNA fragmentation pattern with more potent effect upon the treatment with carbohydrates. Ketoprofen groups showed no sign of auto-recovery by time.

\section{Liver histopathological analysis}

Liver section of control and control-treated rats with plant carbohydrate and oil showed preserved hepatic lobular architecture and structure with more pronounced effect to plant seeds carbohydrate treatment (Fig. 3a-c). Ketoprofen group showed moderate diffuse hydropic degeneration, vacuolation, congested sinusoids, congested central vein, and scattered multinucleated hepatocytes (Fig. 3d). Ketoprofen rats treated with carbohydrate and oil as well as silymarin showed preserved hepatic lobular architecture and structure (Fig. 3e-g).

Table 7 Therapeutic effect of $P$. ovata seed carbohydrates and fixed oil on DNA fragmentation indices of liver tissue in ketoprofen toxicity rats

\begin{tabular}{|c|c|c|c|c|c|}
\hline Groups & $\%$ Tailed DNA & $\%$ Untailed DNA & DNA tail length $(\mu \mathrm{m})$ & $\%$ Total DNA & Tail moment \\
\hline Control & 5.00 & 95.00 & $1.35^{f} \pm 0.13$ & 1.21 & 1.63 \\
\hline P. ovata seed carbohydrates & 4.00 & 96.00 & $1.33^{f} \pm 0.05$ & 1.23 & 1.64 \\
\hline P. ovata seeds oil & 5.00 & 95.00 & $1.29^{f} \pm 0.03$ & 1.27 & 1.64 \\
\hline Ketoprofen & 16.00 & 84.00 & $2.85^{\mathrm{a}} \pm 0.04$ & 2.79 & 7.95 \\
\hline $\begin{array}{l}\text { Ketoprofen }+ \\
\text { P. ovata seed carbohydrates }\end{array}$ & 11.00 & 89.00 & $2.31^{d} \pm 0.08$ & 2.24 & 5.17 \\
\hline $\begin{array}{l}\text { Ketoprofen }+ \\
\text { P. ovata seeds oil }\end{array}$ & 13.00 & 87.00 & $2.59^{b} \pm 0.02$ & 2.51 & 6.50 \\
\hline $\begin{array}{l}\text { Ketoprofen + } \\
\text { Silymarin. }\end{array}$ & 9.00 & 91.00 & $2.11^{e} \pm 0.06$ & 2.05 & 4.33 \\
\hline Ketoprofen left 1 week for recovery & 14.00 & 86.00 & $2.48^{\mathrm{abc}} \pm 0.04$ & 2.44 & 6.05 \\
\hline Ketoprofen left 2 weeks for recovery & 14.00 & 86.00 & $2.57^{\mathrm{ab}} \pm 0.02$ & 2.49 & 6.35 \\
\hline Ketoprofen left 3 weeks for recovery & 12.00 & 88.00 & $2.42^{\mathrm{abcd}} \pm 0.02$ & 2.31 & 5.59 \\
\hline
\end{tabular}

Values are \% of the total counts in each assay

Tail Moment (unit) $=$ tail length $x \%$ total DNA

Data in DNA tail length are expressed as mean \pm SD of three reading

Groups having the same letters are non-significant while others having different letters are significant at $p<0.05$ 

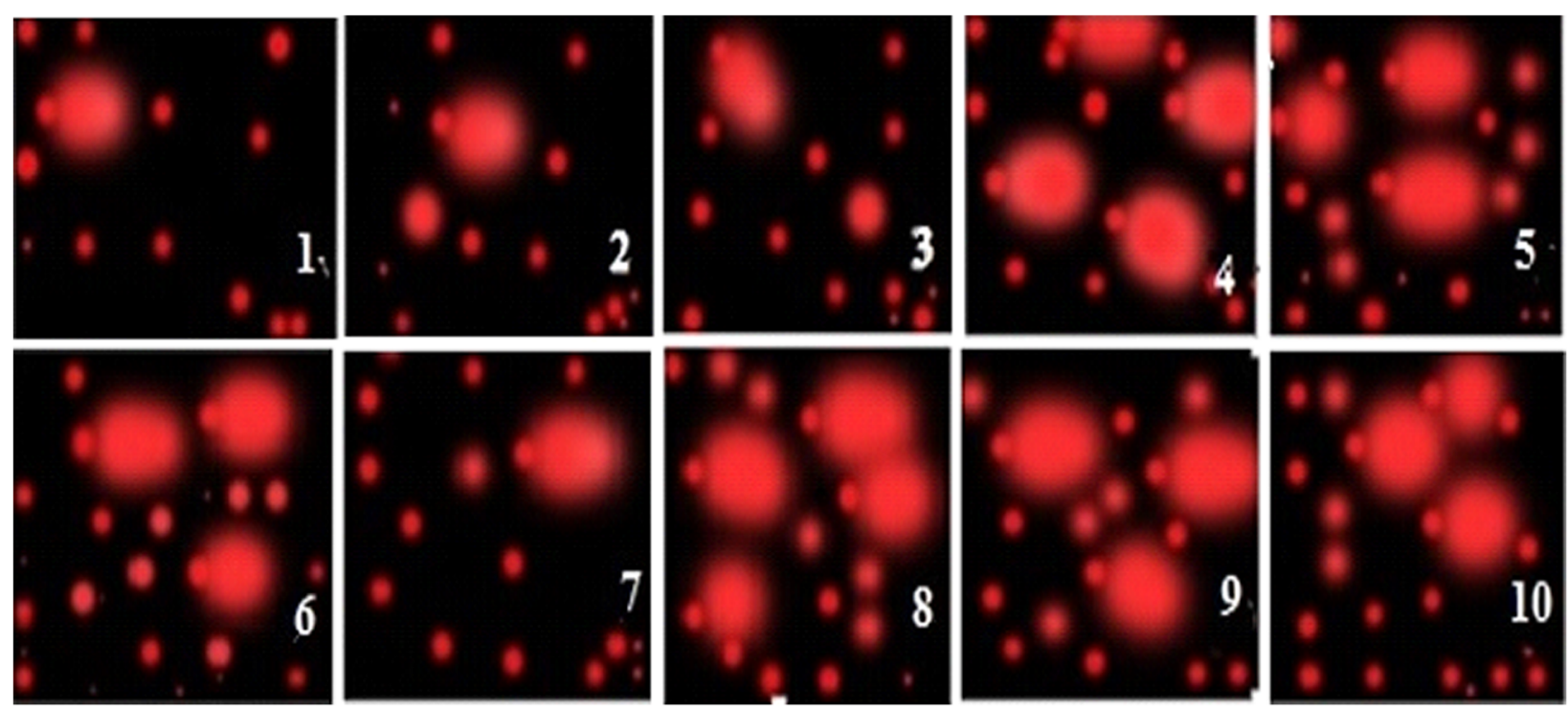

Fig. 2 DNA fragmentation pattern in liver cells of rats after different treatments. Slide (1): control group with no significant of DNA damage. Slide (2 and 3): P. ovata seed carbohydrates and fixed oil groups with non significant DNA damage. Slide (4): ketoprofen group with remarkable percent of DNA damage. Slides $(5,6$, and 7$)$ : ketoprofen rats treated with $P$. ovata seed carbohydrates, $P$. ovata seed oil, and silymarin reference drug respectively, show lesser percent of DNA damage relative to ketoprofen group. Slides (8, 9, and 10): self recovery groups for 1 week, 2 weeks, and 3 weeks respectively with lesser percent of DNA damage relative to ketoprofen group. It is important to note that, $P$. ovata seed carbohydrates showing the most significant reduction in the percent of DNA damage

\section{Kidney function indices}

With respect to kidney function parameters, insignificant changes were observed in creatinine, urea and total protein levels after administration of normal rats with plant seed oil and carbohydrates. Rats administered with ketoprofen showed significant increase in their levels as compared with the control group. Significant decrease in creatinine, urea and total protein levels was reported in ketoprofen-induced rats treated with plant seeds oil and carbohydrates as compared by the ketoprofen group (Table 8). Treatments with plant seeds carbohydrate, seeds oil, and silymarin showed amelioration in urea level by $108.75,103.10$, and $94.82 \%$, respectively, while creatinine level showed improvement by 131.19 , 109.74 , and $127.85 \%$, respectively. In addition, total protein content recorded improvment by 16.61, 12.88 and $14.23 \%$, respectively. A positive correlation was noticed between these parameters in the auto-recovery rats with time (Fig. 1c).

\section{Kidney histopathological analysis}

Kidney of control and control treated rats showed renal corpuscle with normal glomerulus and normal pattern of proximal convoluted and distal tubules, where treatment with carbohydrate recorded the most pronounced effect (Fig. 4a-c). Kidney of ketoprofen-induced rats showed collapsed and atrophic glomeruli corpuscles (Fig. 4d). Ketoprofen rats treated with carbohydrate, oil, and silymarin showed renal cortex and renal corpuscle with almost normal glomerulus and proximal convoluted and distal tubules (Fig. 4e-g).

\section{Liver and kidney histopathological recovery}

Recovered liver for 1-3 weeks recorded preserved hepatic lobular architecture and moderate diffuse hydropic degeneration and vacuolation (Fig. 5a-c). Recovered kidneys for 1-3 weeks showed hyperplasia of epithelial cells lining the partial layer of Bowman's capsule with variable degrees (Fig. 5d-f).

\section{Discussion}

The present study proved the presence of a good variety of secondary metabolites in the polar and nonpolar extracts of $P$. ovata seeds, where the aqueous extract is rich in many sugars, while the petroleum ether extract contain several saturated, unsaturated fatty acids, hydrocarbons, steroids, and terpenoids compounds. Some of these compounds were detected before from the seed such as $\alpha$ and $\gamma$-linolenic acids which are well known for their nutritional values (Gill and Valivety 1997).

The use of certain plants with high concentrations of antioxidant compounds has implied an efficient curative advance against hepatic and oxidative damage (Embuscado 2015; Mishra et al. 2015). P. ovata seeds have high total phenolic content with great antioxidants and DPPH scavenging activity than leaves (Patel et al. 2016). Moreover, some triterpenoids that were isolated from the leaf wax of Plantago spp. such as oleanolic, ursolic, 18 

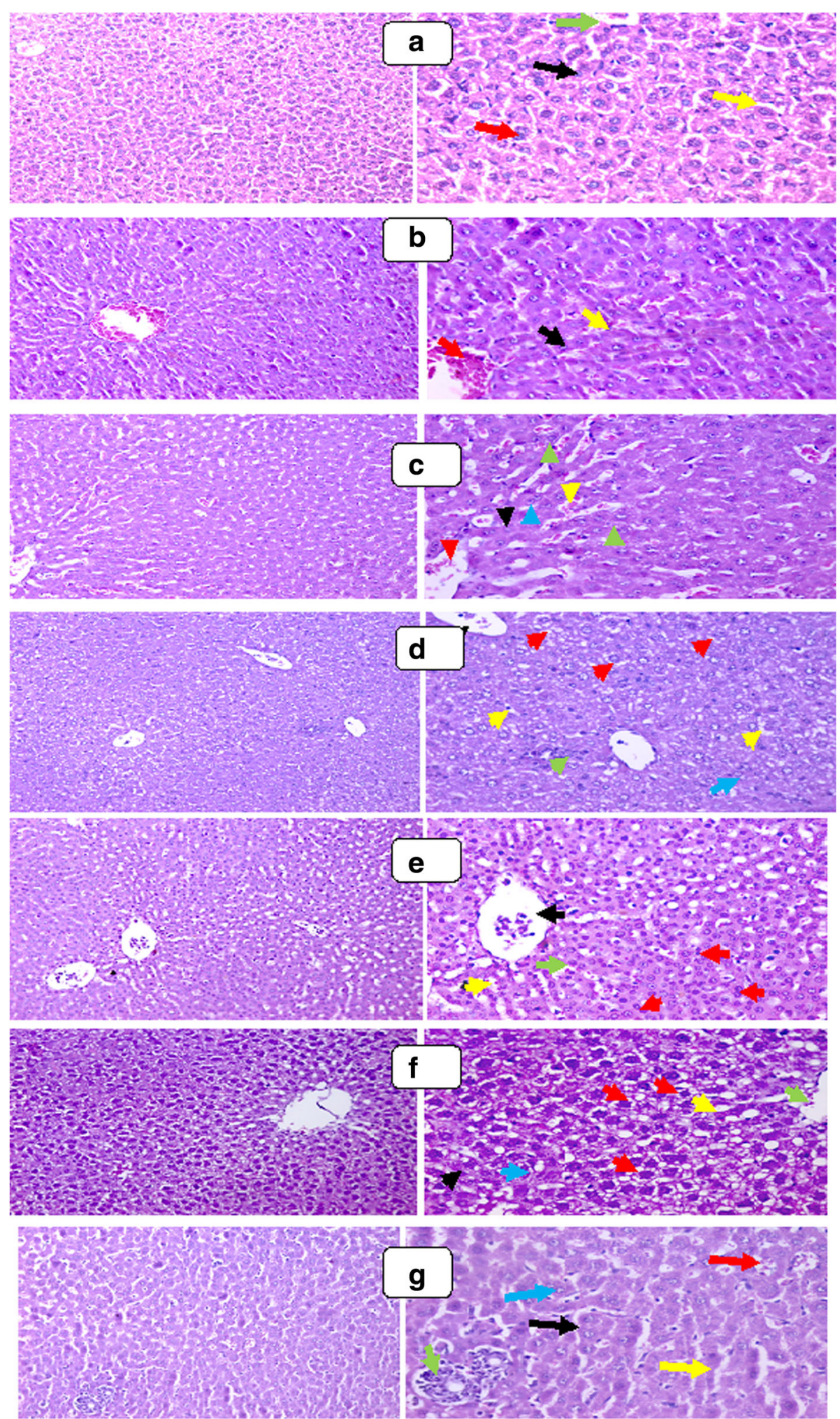

Fig. 3 (See legend on next page.) 
(See figure on previous page.)

Fig. 3 a Liver section from control rat showed normal hepatic structure. b Control rats treated with plant seeds carbohydrate showed preserved hepatic lobular architecture, hepatocytes arranged in thin plates (black arrow), dilated congested sinusoids (yellow arrow), and congested central vein (red arrow). c Control rats treated with plant seeds oil showed preserved hepatic lobular architecture, hepatocytes arranged in thin plates (black arrow) with cytoplasmic vacuoles (green arrow), scattered multinucleated hepatocytes (blue arrow), dilated congested sinusoids (yellow arrow), and congested central vein (red arrow). $\mathbf{d}$ Liver section of ketoprofen induced rats showed hepatocytes with moderate diffuse hydropic degeneration and vacuolation (non-fatty type) (red arrows), congested sinusoids (yellow arrow), congested central vein (black arrow), and scattered multinucleated hepatocytes (blue arrow). e Liver section of ketoprofen rats treated with carbohydrate showed preserved hepatic lobular architecture and structure, hepatocytes arranged in thin plates (green arrow) and dilated congested sinusoids (yellow arrow), congested central vein (black arrow), and many multinucleated hepatocytes (blue arrow). f Liver section of ketoprofen rats treated with plant oil showed preserved hepatic lobular architecture, hepatocytes arranged in thin plates (black arrow), hepatocytes showed hydropic degeneration cytoplasmic vacuoles (non fatty type) (red arrow), scattered multinucleated hepatocytes (blue arrow), dilated congested sinusoids (yellow arrow), and congested central vein (green arrow). $\mathbf{g}$ Liver section of ketoprofen rats treated with silymarin showed preserved hepatic lobular architecture, hepatocytes arranged in thin plates (black arrow), mild hydropic changes (yellow arrows), scattered multinucleated hepatocytes (green arrow), and dilated congested sinusoids (red arrow) $(H \& E, \times 200, \times 400)$

$\beta$-glycyrrhetinic acids, and sitosterol (Ringbom et al. 1998) proved to have many biological activities, as antihypercholesterolemic (Anderson et al. 1990 and Atta et al. 2006) and anti-diabetic agents (Ahmed et al. 2010). In addition, it was found that sterol-containing extracts have a significant inhibition on superoxide generation and elastase release by activated neutrophils, indicating anti-inflammatory and antioxidative actions (Alam et al. 2016). Previous studies demonstrated that $\beta$-sitosterol increased the activities of antioxidant enzymes as superoxide dismutase and glutathione peroxidase in macrophages under oxidative stress (Vivancos and Moreno
2005). Treatment of $\beta$-sitosterol and campesterol significantly inhibit various types of cancer (Awad et al. 2001; De Stefani et al. 2000).

Plant polysaccharides possessed antioxidant and anticancer activities (Jiao et al. 2016). It has been reported that the antioxidant effect of a compound may be concomitant with the development of reducing power. The reducing properties were generally associated with the presence of reductones, which have been shown to exert antioxidant action by breaking free radical chain through donating the hydrogen atom (Zeng et al. 2016). The plant polysaccharides

Table 8 Therapeutic effect of $P$. ovata seed carbohydrates and fixed oil on kidney function indices of ketoprofen toxicity in rats

\begin{tabular}{|c|c|c|c|}
\hline Groups & Urea (mg/dl) & Creatinine $(\mathrm{mg} / \mathrm{dl})$ & Protein $(\mathrm{g} / \mathrm{L})$ \\
\hline Control & $\begin{array}{l}43.50^{\mathrm{d}} \pm 3.80 \\
-\end{array}$ & $\begin{array}{l}3.59^{9} \pm 0.28 \\
-\end{array}$ & $\begin{array}{l}73.75^{c d} \pm 2.63 \\
-\end{array}$ \\
\hline P. ovata seed carbohydrates & $\begin{array}{l}47.40^{d} \pm 4.68 \\
(+8.96)\end{array}$ & $\begin{array}{l}4.15^{\mathrm{fg}} \pm 0.29 \\
(+15.60)\end{array}$ & $\begin{array}{l}70.25 \text { de } \\
(-4.74)\end{array}$ \\
\hline P. ovata seeds oil & $\begin{array}{l}54.06^{d} \pm 4.68 \\
(+24.27)\end{array}$ & $\begin{array}{l}4.40^{f} \pm 0.16 \\
(+22.56)\end{array}$ & $\begin{array}{l}65.75^{\text {de }} \pm 4.39 \\
(-10.83)\end{array}$ \\
\hline Ketoprofen & $\begin{array}{l}127.93^{\mathrm{a}} \pm 9.14 \\
(+194.10)\end{array}$ & $\begin{array}{l}11.93^{\mathrm{a}} \pm 0.26 \\
(+232.31)\end{array}$ & $\begin{array}{l}92.25^{\mathrm{a}} \pm 4.57 \\
(+25.08)\end{array}$ \\
\hline $\begin{array}{l}\text { Ketoprofen }+ \\
\text { P. ovata seed carbohydrates }\end{array}$ & $\begin{array}{l}80.62^{c} \pm 5.51 \\
{[-36.98]}\end{array}$ & $\begin{array}{l}7.22^{\mathrm{e}} \pm 0.81 \\
{[-39.48]}\end{array}$ & $\begin{array}{l}80.50^{b c} \pm 3.70 \\
{[-12.73]}\end{array}$ \\
\hline $\begin{array}{l}\text { Ketoprofen }+ \\
\text { P. ovata seeds oil }\end{array}$ & $\begin{array}{l}83.08^{\mathrm{C}} \pm 5.16 \\
{[-35.06]}\end{array}$ & $\begin{array}{l}7.99^{d} \pm 0.28 \\
{[-33.02]}\end{array}$ & $\begin{array}{l}82.75^{b} \pm 4.96 \\
{[-10.30]}\end{array}$ \\
\hline $\begin{array}{l}\text { Ketoprofen + } \\
\text { Silymarin. }\end{array}$ & $\begin{array}{l}86.68^{c} \pm 5.53 \\
{[-32.24]}\end{array}$ & $\begin{array}{l}7.34^{e} \pm 0.98 \\
{[-38.47]}\end{array}$ & $\begin{array}{l}81.75^{b c} \pm 3.86 \\
{[-11.38]}\end{array}$ \\
\hline Ketoprofen left 1 week for recovery & $\begin{array}{l}119.48^{\mathrm{a}} \pm 9.48 \\
{[-6.60]}\end{array}$ & $\begin{array}{l}11.46^{\mathrm{ab}} \pm 0.41 \\
{[-3.93]}\end{array}$ & $\begin{array}{l}89.25^{\mathrm{ab}} \pm 3.95 \\
{[-3.25]}\end{array}$ \\
\hline Ketoprofen left 2 weeks for recovery & $\begin{array}{l}98.31^{b} \pm 6.22 \\
{[-23.15]}\end{array}$ & $\begin{array}{l}11.05^{b} \pm 0.48 \\
{[-7.37]}\end{array}$ & $\begin{array}{l}84.50^{b} \pm 3.70 \\
{[-8.40]}\end{array}$ \\
\hline Ketoprofen left 3 weeks for recovery & $\begin{array}{l}96.18^{\mathrm{bc}} \pm 5.42 \\
{[-24.81]}\end{array}$ & $\begin{array}{l}8.65^{c} \pm 0.36 \\
{[-27.49]}\end{array}$ & $\begin{array}{l}81.50^{b c} \pm 6.75 \\
{[-11.65]}\end{array}$ \\
\hline
\end{tabular}

Data are mean \pm SD of six rats/group

Groups having the same letters are non- significantly different, while those having different letters are significantly different at $p<0.05$

Values between brackets are \% changes over control group

Values between parentheses are $\%$ changes over ketoprofen group 


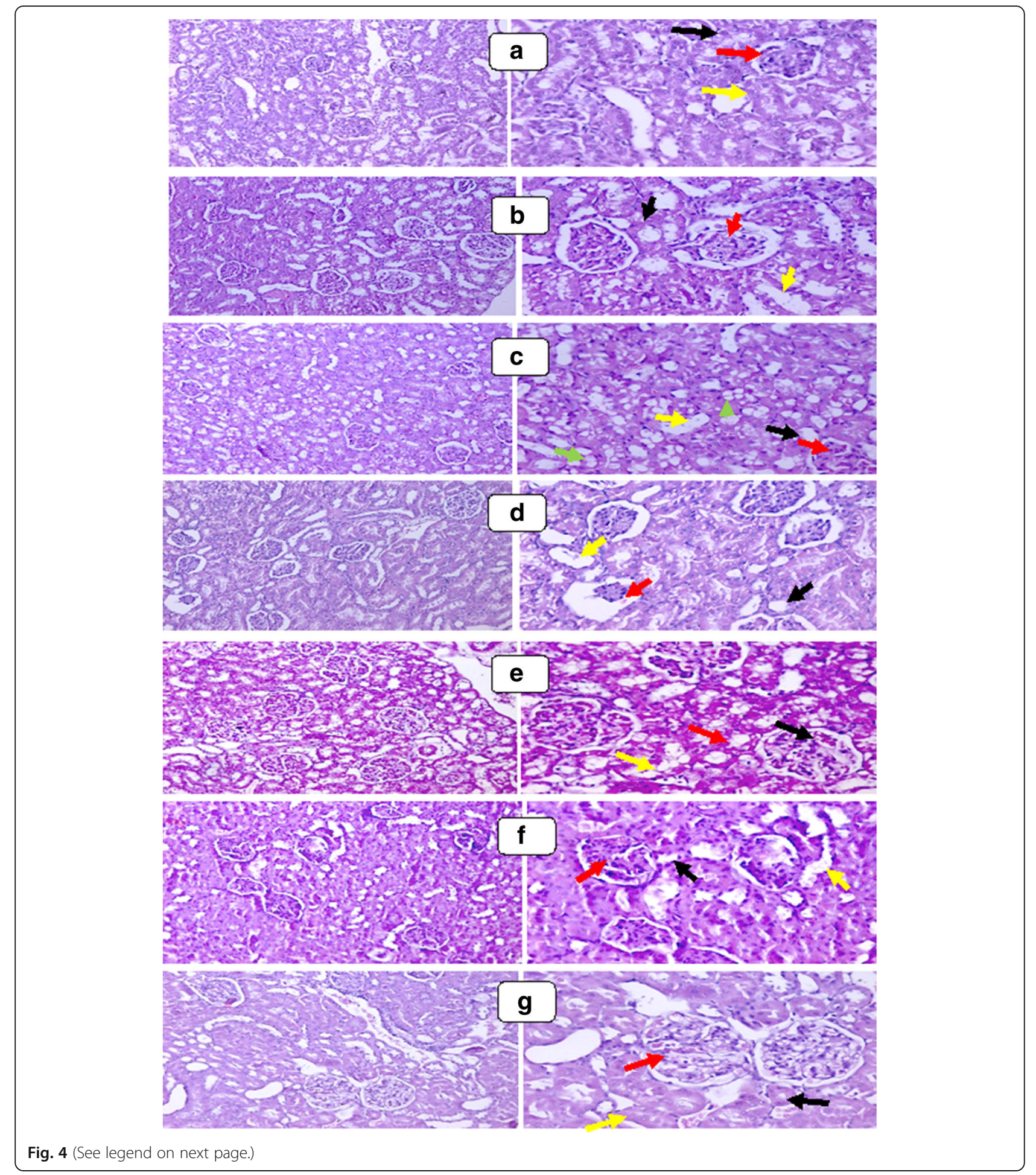

acted as a reducing agent by effecting the conversion of the $\mathrm{Fe} 3+/$ ferricyanide complex to the $\mathrm{Fe} 2+$ form. Metal chelating activity is considered as one of the antioxidant mechanisms, since it reduces the concentration of the transition metal catalyzing lipid peroxidation process (Qiao et al. 2009). Plant polysaccharides have liver cell regenerative effects and are able to stabilize membrane structures in hepatic injury. Therefore, it may give great health benefits to humans and animals with hepatic injuries. 
(See figure on previous page.)

Fig. 4 a Kidney section of control rats showed normal glomeruli. b Kidney section of control rats treated with plant seeds carbohydrate showed renal cortex and renal corpuscle with normal glomerulus (red arrow), normal pattern of proximal convoluted (black arrow), and distal convoluted (yellow arrow) tubules. c Kidney section of control rats treated with plant seeds oil showed few of the glomeruli corpuscles are collapsed and atrophic (red arrow). Proximal convoluted tubules showed destructed epithelial lining (black arrow), destructed epithelial lining of distal convoluted tubules (yellow arrow). d Kidney section of ketoprofen-induced rats showed few of glomeruli corpuscles (hyperplasia of epithelial cells lining the partial layer of Bowman's capsule) (red arrow), proximal and distal convoluted tubules with destruction of the epithelial lining cells (black and yellow arrow), many interstitial epithelial cells with cytoplasmic vacuoles and eccentric nuclei (green arrow). e Kidney section of ketoprofen rats treated with plant seed carbohydrate showed renal cortex and renal corpuscle with almost normal glomerulus (red arrow), almost normal pattern of proximal convoluted (black arrow), and distal convoluted (yellow arrow) tubules. $\mathbf{f}$ Kidney section of ketoprofen rats treated with plant seeds oil showed renal cortex showing renal corpuscle with normal glomerulus (red arrow), normal pattern of proximal convoluted (black arrow), and distal convoluted (yellow arrow) tubules. g Kidney section of ketoprofen rats treated with silymarin showed renal cortex and renal corpuscle with almost normal glomerulus (red arrow), normal pattern of proximal convoluted (black arrow), and distal convoluted (yellow arrow) tubules $(H \& E, \times 200, \times 400)$

Silymarin, an effective extract obtained from seeds of milk thistle (Silybum marianum), is widely used in treatment of different liver diseases (Shaarawy et al. 2009). Silymarin is composed of flavonolignans, flavonoids, fatty acids, and polyphenolic compounds that have different biological activities (Saller et al. 2007). Zhang et al. (2013) reported that it acts as a strong free radical scavenger without any adverse side effects. Moreover, silymarin is described to be an immunomodulatory, antiproliferative, antifibrotic, and antiviral agent. Hence, Freitag et al. (2015) reported that silymarin is used as a reference drug in evaluation the new hepatoprotective drugs by inhibiting lipid peroxidation process. In the present study, MDA, as lipid peroxidation product, recorded significant increase in hepatic tissue of ketoprofen-induced hepatotoxicity in rats. We can determine oxidative stress by increased ROS and the antioxidant systems including SOD and glutathione, which act as protectors of oxidative stress. Hence, silymarin, by reducing the free radical load, is probably able to antagonize the depletion of the two main detoxifying mechanisms (Fraschini et al. 2002). In addition, Motawi et al. (2016) and (2017) mentioned the role of free radicals in the fragmentation pattern of DNA.

In our study, treatment with silymarin and plant seeds carbohydrate and oil restored the levels of AST, ALT, and ALP to normal values, and decreased MDA level, which alleviates deleterious effects induced by ketoprofen. In accordance with our study, Shalan et al. (2005) mentioned that silymarin has a protective effect against experimental hepatotoxicity by regulating the actions of the ultra-structures of the liver cells.

In addition, Romero et al. (1998) showed that liver intoxication-induced changes in protein synthesis process. Hence, the observed increase in total protein as clearly shown in our studies can be deemed as an index of cellular dysfunction in liver and kidney diseases. The most commonly associated characteristic of liver fibrosis is the increased deposition of collagens. During liver fibrosis, altered collagen synthesis or deposition at both mRNA and protein levels is observed which give us an additional support to the observed fragmentation of DNA (Motawi et al. 2011).

Renal function may be affected through inhibition of renal prostaglandins synthesis by non-steroidal anti-inflammatory drug (Farag Allah 2001). This is because prostaglandins are involved in the regulation of solute homeostasis, glomerular filtration, and vascular tone, which are vital processes for normal kidney function. Urea and creatinine are the most metabolic waste products that are freely filtered by the glomeruli (Aprioku and Uche 2013). In most clinical and toxicological investigations, their serum concentrations are commonly used as potential markers of renal toxicity (Perrone et al. 1992). The antioxidant action of resveratrol, tyrosol, and b-sitosterol increase prostaglandin E2 synthesis in stimulated macrophages (Vivancos and Moreno 2005 and 2008). This observation explained the therapeutic role of $P$. ovata in renal injury through the stimulation of prostaglandin level, suppression of free radicals and amelioration of the antioxidant levels which will be reflected to the ultra-structures of kidney cells, glomeruli and tubules.

\section{Conclusion}

Carbohydrate and fixed oil extracted from Plantago ovata L. seeds succeeded to treat the hepatorenal toxicity in rats with potent effect of carbohydrate. Hepatorenal toxicity is an irreversible mechanism, where the auto-healing percentage not showed a significant concern. Further works are needed to fully characterize these polysaccharides and elucidate its possible mode of action. 

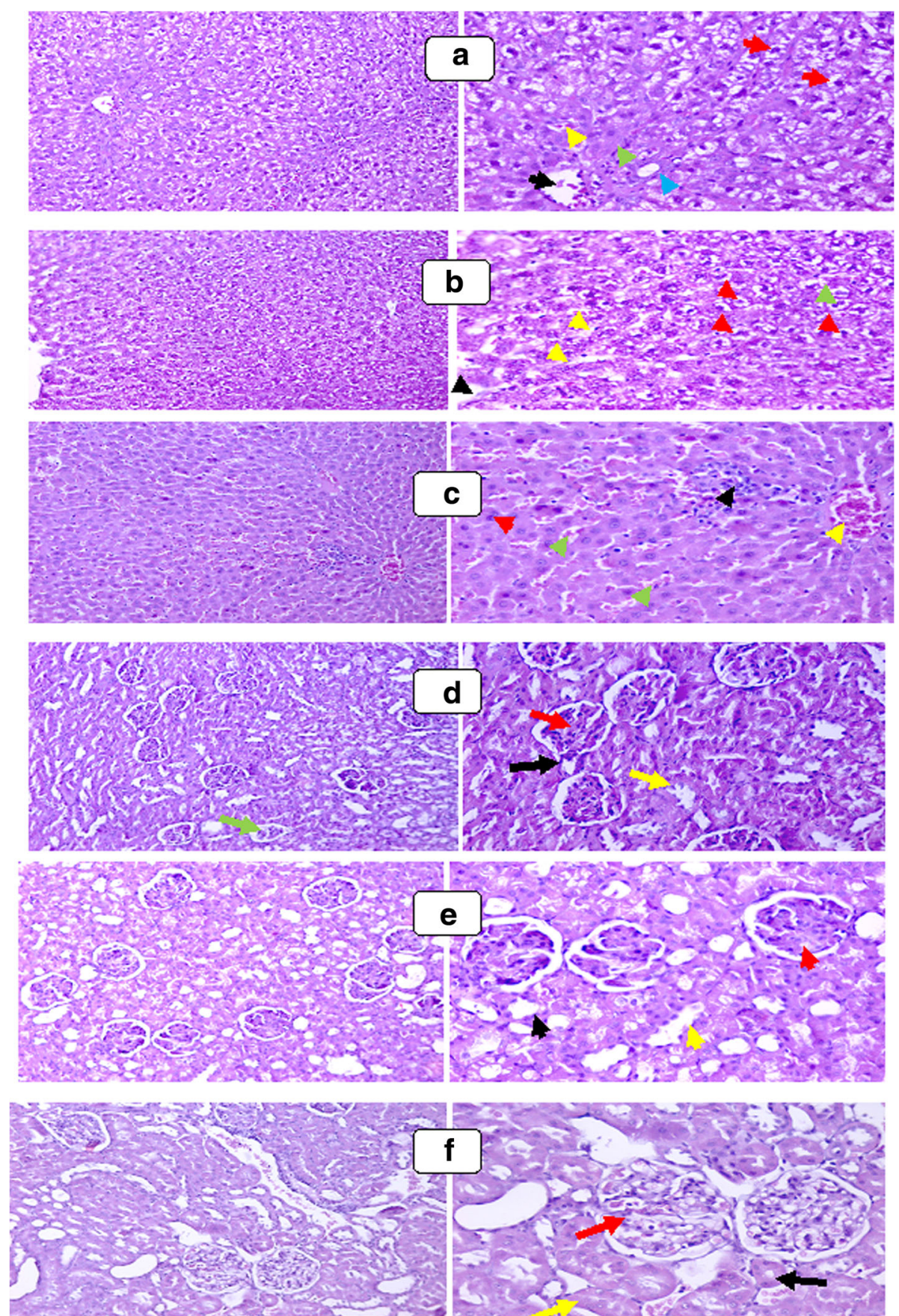

Fig. 5 a-c Liver section of ketoprofen rats left free without treatment for different durations (1-3 weeks) showed preserved hepatic lobular architecture, moderate diffuse hydropic degeneration and vacuolation (non-fatty type)(red arrows), congested sinusoids (yellow arrow), congested central vein (black arrow), scattered multinucleated hepatocytes (blue arrow), and mild infiltration of inflammatory (green arrow). $\mathbf{d}-\mathbf{f}$ Kidney section from auto-recovery groups for 1-3 weeks showed few of glomeruli corpuscles (hyperplasia of epithelial cells lining the partial layer of Bowman's capsule) (red arrow) with atrophy (green arrow), proximal and distal convoluted tubules with mild destructed epithelial lining cells (black and yellow arrow) $(H \& E, \times 200, \times 400)$ 


\section{Acknowledgements}

We would like to acknowledge the National Research center for supporting and facilitating our work.

\section{Funding}

Not applicable.

\section{Availability of data and materials}

Not applicable.

\section{Authors' contributions}

AME and MME designed the plant experiments, did all the tests of isolation and identification of the plant bioactive compounds, and wrote this section. AFA participated with MAH in designing the biological experiments. AFA did all the biochemical experiments and the statistical analysis of the biological data. All authors contributed in manuscript draft writing. MAH revised the final form of the article and approved it. All authors read and approved the final manuscript.

\section{Ethics approval and consent to participate}

The study was complied with the ethical guidelines of Medical Ethical Committee of the National Research Center in Egypt Approval No. 18211.

\section{Consent for publication}

Not applicable.

\section{Competing interests}

The authors declare that they have no competing interests.

\section{Publisher's Note}

Springer Nature remains neutral with regard to jurisdictional claims in published maps and institutional affiliations.

\section{Author details}

${ }^{1}$ Pharmacognosy Department, National Research Centre, 33El-Bohouth St. Dokki, Giza, Egypt. ${ }^{2}$ Department of Therapeutic Chemistry, National Research Centre, 33, El-Bohouth St., Dokki, Giza, Egypt.

Received: 17 October 2018 Accepted: 11 November 2018 Published online: 28 November 2018

\section{References}

Adams P (1989) Identification of fixed oils by ion trap mass spectroscopy. Academic Press, INC, New York

Ahmed I, Naeem M, Shakoor A, Ahmed Z, Muhammad H, labal N (2010) Investigation of anti-diabetic and Hypocholesterolemic potential of psyllium husk fiber (Plantago psyllium) in diabetic and hypercholesterolemic albino rats. World Acad Sci Eng Technol 37:1229-1233

Alam A, Ferdosh S, Ghafoor K, Hakim A, Juraimi AS, Khatib A et al (2016) Clinacanthus nutans: a review of the medicinal uses, pharmacology and phytochemistry. Asian Pac J Trop Med 9:402-409

Anderson JW, Deakins DA, Floore TL, Smith BM, Whitis SE (1990) Dietary fiber and coronary heart disease. Crit Rev Food Sci Nut 29:95-147

Aprioku JS, Uche FI (2013) Renal effects of non-steroidal anti inflammatory drugs in albino rats. Br J Pharmaceut Res 3:314-325

Atta AH, Nasr SM, Mouneir SM (2006) Potential protective effect of some plant extracts against carbon tetrachloride-induced hepatotoxicity. Afr J Tradit Complement Altern Med 3:1-9

Awad AB, Williams H, Fink CS (2001) Phytosterols reduce in vitro metastatic ability of MDA-MB-231 human breast cancer cells. Nutr Cancer 40:157-164

Awad NE, Seida AA, Hamed MA, Elbatanony MM (2011) Hypolipidaemic and antioxidant activities of Ficus microcarpa (L.) in hypercholesterolemic rats. Nat Prod Res 25:1202-1207

Bartels H, Böhmer M (1971) Einemikro method zurkreatinin-bestimmung. Clin Chim Acta 32:81-85

Beara IN, Lesjak MM, Jovin ED, Balog KJ, Anackov GT, Orcic DZ, Dukic NM (2009) Plantain (Plantago L.) species as novel sources of flavonoid antioxidants. J Agric Food Chem 57:9268-9273

Beara IN, Orcic DZ, Lesjak MM, Mimica-Dukic NM, Pekovic BA, Popovic MR (2010) Liquid chromatography/ tandem mass spectrometry study of antiinflammatory activity of plantain (Plantago L.) species. J Pharm Biomed Anal 52:701-706
Bradford MM (1976) A rapid and sensitive method for the quantitation of microgram quantities of protein utilizing the principle of protein-dye binding. Anal Biochem 72:248-254

Buege JA, Aust SD (1978) Microsomal lipid peroxidation. Methods Enzymol 52: 302-310

Chiang LC, Chiang W, Chang MY, Lin CC (2003) In vitro cytotoxic: antiviral and immunomodulatory effects of Plantago major and Plantagoasiatica. Am J Chinese Med 31:225-234

Choi JM, Lee EO, Lee HJ, Kim KH, Ahn KS, Shim BS, Kim NI, Song MC, Baek NI, Kim SH (2007) Identification of Campesterol from Chrysanthemum coronarium L. and its antiangiogenic activities. Phytother Res 21:954-959

De Stefani E, Boffetta P, Ronco AL, Brennan P, Deneo-Pellegrini H, Carzoglio JC et al (2000) Plant sterols and risk of stomach cancer: a case-control study in Uruguay. Nutr Cancer 37:140-144

Dubois M, Gilles KA, Hamilton JK, Rebers PA, Smith F (1956) Colorimetric method for determination of sugars and related substances. Anal Chem 28:350-356

Embuscado ME (2015) Spices and herbs: natural sources of antioxidants - a mini review. J Funct Foods 18:811-819

Evans W (1962) Pharmacognosy, 3rd edn. Balilliene Tindall, London

Farag Allah AM (2001) The side effects of the nonsteroidal anti-inflammatory drug (NSAID) ketoprofen on histological and ultrastructural aspects of the kidneys of albino rats. Egypt J Hospital Med 3:161-176

Fraschini F, Demartini G, Esposti D (2002) Pharmacology of silymarin. Clin Drug Invest 22:51-65

Freitag AF, Cardia GF, da Rocha BA, Aguiar RP, Silva-Comar FM, Spironello RA et al (2015) Hepatoprotective effect of silymarin (Silybum marianum) on hepatotoxicity induced by acetaminophen in spontaneously hypertensive rats. Evid Based Complement Alternat Med 2015:538317

Gella FJ, Olivella T, Cruz PM, Arenas J, Moreno R, Durban R, Gomez JAA (1985) Simple procedure for routine determination of aspartate aminotransferase and alanine aminotransferase with pyridoxalphosphate. Clin Chem Acta 153: 241-247

Gertz CH (1990) HPLC Tips and Tricks. LDC analytical, Riviera Beach, p 608

Gill I, Valivety R (1997) Polyunsaturated fatty acids, part1: occurrence, biological activities and applications. Trends Botechnol 15:401-409

Haddadian K, Haddadian K, Zahmatkash M (2014) A review of Plantago plant. Ind J Trad Knowledge 13:681-685

Hamed MA, Ali SA, El-Rigal NS (2012) Therapeutic potential of ginger against renal injury induced by carbon tetrachloride in rats. Sci World J 2012:1-12

Heo SK, Cho J, Cheon JW, Choi MK, Im DS, Kim JJ, Choi YG, Jeon Y, Chung SJ, Shim CK, Kim DD (2008) Pharmacokinetics and pharmacodynamics of ketoprofen plasters. Biophann Drug Dispos 29:37-44

Jain PS, Bari SB (2010) Isolation of lupeol, stigmasterol and campesterol from petroleum ether extract of woody stem of Wrightia tinctoria. Asian J Plant Sci 9:163-167

Jiao R, Liu Y, Gao H, Xiao J, So KF (2016) The anti-oxidant and antitumor properties of plant polysaccharides. Am J Chin Med 44:463-488

Kiem PV, Cuong NX, Nhiem NX, Thu VK, Ban NK, Minh CV, Tai BH, Hai TN, Lee SH, Jang HD, Kim YH (2011) Antioxidant activity of a new C-glycosyl flavone from the leaves of Ficus microcarpa. Bioorganic Med Chem Lett 21:633-637

Laidlow M, Percival V (1950) The chemistry of gum and mucilage. J Chem Soc 1: 531 Rembold Publishing Co. New York

Matz SA (1962) Food Texture. The Avi Publishing Company Inc., West Port, Connecticut

Mishra A, Patel MK, Jha B (2015) Non-targeted metabolomics and scavenging activity of reactive oxygen species reveal the potential of Salicornia brachiataasa functional food. J Funct Foods 13:21-31

Molisch H (1886) The monosaccharides. Publishing House of zechoslovak Academy of Science, Prague, p 872

Moradi J, Abbasipour F, Zaringhalam J, Maleki B, Ziaee N, Khodadoustan A, Janahmadi M (2014) A medicinal plant compound, decreases the production of pro-inflammatory TNF- $\alpha$ and IL-1 $\beta$ in a rat model of LPS-induced periodontitis. Iranian J Pharmaceut Res 13:1319-1325

Moron MS, Depierre JW, Mannervik B (1979) Level of glutathione, glutathione reductase and glutathione-S-transferase activities in rat lung and liver. Biochim Biophys Acta 582:67-78

Motawi TK, Ahmed SA, Hamed MA, El-Maraghy SA, Aziz WM (2016) Combination of melatonin and certain drugs for treatment of diabetic nephropathy in streptozotocin induced diabetes in rats. Diabatol Internat 7:413-424

Motawi TK, Darwish HA, Hamed MA, El-Rigal NS, Aboul Naser AF (2017) Coenzyme Q10 and niacin mitigate streptozotocin-induced diabetic encephalopathy in a rat model. Metab Brain Dis 32:1519-1527 
Motawi TK, Hamed MA, Shabana MH, Hashem RM, Aboul Naser AF (2011) Zingiber officinale acts as a nutraceutical agent against liver fibrosis. Nutr Metab 8:40-50

Nishikimi M, Rae NA, Yagi K (1972) The occurrence of superoxide anion in the action of reduced phenazinemethosulphate and molecular oxygen. Biochem Biophys Res Commun 46:849-853

Pascal S, Taton M, Rahier A (1993) Plant sterol biosynthesis. Identification and characterization of two distinct microsomal oxidative enzymatic systems involved in sterol c4-demethylation. J Biol Chem 268:11639-11165

Patel MK, Mishra A, Jha B (2016) Non-targeted metabolite profiling and scavenging activity unveil the nutraceutical potential of Psyllium (Plantago ovata Forsk). Plant Sci 7. https://doi.org/10.3389/fpls.2016.00431

Perrone R, Madias N, Levy A (1992) Serum creatinine as an index of renal function: new insights into old concepts. Clin Chem 38:1933-1953

Qiao D, Ke C, Hu B, Luo J, Ye H, Sun Y, Yan X, Zeng X (2009) Antioxidant activities of polysaccharides from Hyriopsis cumingii. Carbohydr Polym 78:199-204

Ringbom T, Segura L, Noreen Y, Perera P, Bolin L (1998) Ursolic acid from Plantago major, a selective inhibitor of cyclooxygenase-2 catalyzed prostaglandin biosynthesis. J Nat Prod 61:1212-1215

Romero FJ, Bosch-Morell F, Romero MJ, Jareno EJ, Romero B, Marín N, Romá J (1998) Lipid peroxidation products and antioxidants in human disease. Environ Health Perspect 106:1229-1234

Ronsted N, Franzyk H, Molgaard P, Jaroszewski JW, Jensen SR (2003) Chemotaxonomy and evolution of Plantago L. Plant Syst Evol 242:63-82

Rosalki SB, Foo AY, Burlina A (1993) Multicenter evaluation of iso-ALP test kit for measurement of bone alkaline phosphatase activity in serum and plasma. Clin Chem 39:648-652

Saller R, Melzer J, Reichling J, Brignoli R, Meier R (2007) An updated systematic review of the pharmacology of silymarin. Forsch Komplement Med 14(2):70-80

Samuelsen AB (2000) The traditional uses, chemical constituents and biological activities of Plantago major L. A review. J Ethnopharmacol 711:21

Shaarawy SM, Tohamy AA, Elgendy SM, Abd Elmageed ZY, Bahnasy A, Mohamed MS et al (2009) Protective effects of garlic and silymarin on NDEA-induced rats hepatotoxicity. Int J Biol Sci 5:549-557

Shalan MG, Mostafa MS, Hassouna MM, Hassab El-Nabi SE, El-Refaie A (2005) Amelioration of lead toxicity on rat liver with vitamin $C$ and silymarin supplements. Toxicology 206:1-15

Stahl E (1969) Thin layer chromatography a laboratory HandBook, 2nd edn. Springer Verlag, Berlin, Heidelberg, NewYork

Subraya CK, Satyanarayana D, Yamini D (2013) Isolation and characterization of steroids and a triterpenoid from Phyllanth uslawii. Der Pharma Chemica 5:63-67

Suzuki H, Suzuki K (1998) Rat hypoplastic kidney (hpk/hpk) induces renal anemia, hyperparathyroidism, and osteodystrophy at the end stage of renal failure. J Vet Med Sci 60:1051-1058

Tabacco A, Meiattini F, Moda E, Tarli P (1979) Simplified enzymic/colorimetric serum urea nitrogen determination. Clin Chem 25:336-337

Tomic Z, Milijasevic B, Sabot A, Dusan L, Jevic V, Mikov M, Majda AS, Vasovic V (2008) Diclofenac and ketoprofen liver toxicity in rat. Eur J Drug Metab Pharmacokinetiks 33:253-260

Tsuda K, Sakai K, Tanabe K, Kishida Y (1960) Isolation of 22-dehydrocholestrol from Hypnea japonica. J Am Chem Soc 82:1442-1443

Vivancos M, Moreno JJ (2005) Beta-sitosterol modulates antioxidant enzyme response in RAW 264. 7 macrophages. Free Radic Biol Med 39:91-97

Vivancos M, Moreno JJ (2008) Effect of resveratrol, tyrosol and b-sitosterol on oxidised low-density lipoprotein-stimulated oxidative stress, arachidonic acid release and prostaglandin E2 synthesis by RAW 264.7 macrophages. Br J Nutr 99:1199-1207

Zeng B, Su M, Chen Q, Chang Q, Wang W, Li H (2016) Antioxidant and hepatoprotective activities of polysaccharides from an Oectochilus roxburghii. Carbohydr Polym 153:391-398

Zhang W, Hong R, Tian T (2013) Silymarin's protective effects and possible mechanisms on alcoholic fatty liver for rats. Biomol Ther 21:264-269

\section{Submit your manuscript to a SpringerOpen ${ }^{\circ}$ journal and benefit from:}

- Convenient online submission

- Rigorous peer review

- Open access: articles freely available online

High visibility within the field

- Retaining the copyright to your article

Submit your next manuscript at $\boldsymbol{\nabla}$ springeropen.com 